\title{
Efectos del Tratamiento de Homogeneización en la Trabajabilidad de la Aleación CuNiSiCr
}

\author{
Ernesto G Maffia ${ }^{(1)}$, Daniel Tovio, Ana L Cozzarin ${ }^{(2)}$ y Alfredo González ${ }^{(2)}$ \\ (1) Universidad Nacional de La Plata, (UNLP), Facultad de Ingeniería de La Plata, Lab. de \\ Investigaciones en Metalurgia Física LIMF, Calle 1 y 47, La Plata-Argentina. \\ (e-mail: ernesto.maffia@ing.unlp.edu.ar) \\ (2) Facultad de Ingeniería de La Plata (UNLP), Conicet, Calle 1 y 47, La Plata-Argentina.
}

Recibido May. 22, 2009; Aceptado Jul. 13, 2009; Versión Final recibida Jun. 24, 2010

\begin{abstract}
Resumen
Con el objeto de mejorar la trabajabilidad de aleaciones CuNiSiCr se realizó un estudio de las condiciones del tratamiento térmico de homogeneizado y se evaluó su comportamiento mecánico a través de ensayos de tracción. Se realizaron ensayos de homogeneizados a 850,950 y $1025^{\circ} \mathrm{C}$ con tiempos de mantenimientos de $0,1,5$ y 6 horas y un posterior doble envejecido. Sobre ese material se realizaron ensayos de tracción a temperatura ambiente. Los resultados muestran una importante mejora en la trabajabilidad para temperaturas de $850^{\circ} \mathrm{C}$ y tiempos de homogeneizados de 6 horas. Esta mejora en la ductilidad está asociada a la presencia de una microestructura conteniendo una escasa cantidad de precipitados discontinuos en límite de grano y precipitados redondeados en el interior de los granos. Se concluye que el tratamiento térmico de homogeneizado es una herramienta adecuada para aumentar la trabajabilidad de la aleación.
\end{abstract}

Palabras clave: aleación CuNiSiCr, tratamiento térmico, homogeneización, crecimiento de grano, ductilidad

\section{Effects of Homogenization Treatment on the Workability of Alloy CuNiSiCr}

\begin{abstract}
In order to improve the workability of alloys CuNiSiCr a study of the conditions of homogenizing heat treatment and assessed their behavior through mechanical tests traction. Standardized tests were conducted at 850,950 and $1025^{\circ} \mathrm{C}$ with time-keeping $0,1.5$ and 6 hours and a subsequent double aged. It was observed that the optimum workability for the alloys occurs at the $850^{\circ} \mathrm{C}$ and 6 hours. This improvement in ductility is associated with the presence of a microstructure containing a small amount of discontinuous precipitates in limiting grain and rounded precipitates inside the grains. It is concluded that the homogenizing heat treatment is and adequate method to increase the workability of the alloy.
\end{abstract}

Keywords: CuNiSiCr alloy, thermal treatment, homogenization, grain growth, ductility 


\section{INTRODUCCIÓN}

En los últimos años se han logrado considerables avances en el desarrollo de materiales no ferrosos observándose una mejora significativa en las propiedades térmicas y eléctricas. Sin embargo, estos materiales no pueden ser utilizados para fines estructurales pues presentan una moderada resistencia mecánica (Apello y Fenici ,1988; Obrutsky et al., 1965; Saarivirta et al., 1960; Susuki et al., 2006)

Actualmente se utilizan para tales aplicaciones aleaciones cobre-berilio, las cuales conjugan alta una resistencia mecánica (1400Mpa), conductividad eléctrica y térmica, mas una optima resistencia a la corrosión y al desgaste. Estas propiedades resultan en materiales aptos para aplicaciones electrónicas y eléctricas, matricería para plásticos entre otras aplicaciones. Sin embargo los humos, nieblas o polvos generados durante su fabricación constituyen un gran factor de riesgo en la salud. Por esta razón y por el elevado costo de fabricación, han surgido algunas opciones de reemplazo, siendo la más promisoria la aleación CuNiSiCr (UNS 18000) (Bhargava y Bhan., 1972; Tewari y Bhan., 1964; Rdzawski y Stobrawa, 1996). Esta aleación se presenta como una alternativa viable, mostrando buenos valores de conductividad eléctrica y térmica así como una buena resistencia mecánica. Sin embargo, durante su procesamiento se han presentado algunas dificultades, lo que advierte sobre la falta de conocimiento de sus propiedades.

Para evaluar esta capacidad para el procesamiento, existe un parámetro que se denomina trabajabilidad (Dieter., 1989). Este concepto expresa la capacidad que posee un material de ser deformado sin fisuras. Si bien el término es difícil de definir totalmente debido a las innumerables variables que aparecen durante el procesamiento de un material, se entiende que mejorar la trabajabilidad de un material significa aumentar su velocidad de procesamiento. Esto se consigue aumentando la ductilidad a través de la aplicación de tratamientos térmicos de homogeneizado.

Diferentes investigadores han estudiado el efecto de los tratamientos térmicos de homogeneizado con la finalidad de aumentar la trabajabilidad de materiales con baja ductilidad. Sokolowsky et al.,(1995), compararon distintos niveles en el tratamiento de solubilizado aplicado en aleaciones de Aluminio A319 en condición como colado. Estos investigadores observaron que la aplicación de este tipo de tratamientos térmicos condujo a una reducción y refinamiento de las segundas fases ricas en cobre y una mejora de la homogeneización de la matriz. Sus resultados también demuestran que las mejoras en la homogeneización conducen a un aumento de la resistencia, ductilidad y energía de impacto. Totik et al.,(2004) estudiaron el efecto de las condiciones del tratamiento térmico de homogeneizado sobre las deformaciones en frío aleaciones AA2014. Sus resultados indican que los tratamientos de homogeneizado convierten las segundas fases, que en principio son grandes y están localizadas en limite de grano, en pequeñas y esparcidas a través de los granos.

La homogenización de un lingote es un tratamiento muy importante para reducir los gradientes de concentración de elementos, antes de los procesos de deformación plástica. Así lo pensaron M. A.Dang-sheng et al (2009) cuando estudiaron los efectos del tratamiento de homogeneizado en la microestructura y la tenacidad al impacto de lingotes de aceros para matrices $\mathrm{H} 13$. Sus resultados muestran que la distancia entre brazos interdendriticos aumenta gradualmente hacia el centro del lingote homogeneizado. Además observaron la eliminación de la segregación en bandas y la transformación mas uniforme de la microestructura, mejorando las propiedades isotropicas del material.

También se observa, en materiales especiales, que el tratamiento de homogeneizado ayuda a optimizar la estructura "as cast" de aleaciones TiAINbWB utilizadas en al industria aeroespacial. Zang Wei et al (2009) estudiaron la optimización de estructuras a través de tratamientos térmico y tratamiento mecánico-térmico para eliminar la fase $\beta$ triaxial (B2). Sus resultados indican que un tratamiento térmico de homogeneizado en la región bifásica $(\alpha+\gamma)$ a $1220^{\circ} \mathrm{C}$ durante 36 horas puede efectivamente eliminar la fase $\beta$. Sin embargo hay un apreciable crecimiento de grano. El aumento de la temperatura de tratamiento térmico $\left(1260^{\circ} \mathrm{C}\right)$ puede reducir el tiempo de mantenimiento (reducido a 8 h) y evitar de manera efectiva el crecimiento de los granos. 
En la actualidad, las aleaciones de magnesio tienen una gran importancia en la industria automovilística de punta debido a bajo peso y alta resistencia especifica, pero poseen una pobre resistencia al creep. J. Zhang et al., (2009) estudiaron la evolución microestructural durante el homogeneizado de la aleación ZnMgAl. Para evaluar el grado de homogeneización con respecto a la distribución microquímica, utilizaron materiales obtenidos después de un tratamiento de homogeneización isotérmica y temple. Los resultados presentados por estos investigadores muestran que para cortos tiempos de mantenimiento durante la homogenización, resultan en lo referente al fenómeno de precipitación in situ, en una difusión insuficiente de $\mathrm{Zn}$ y Al desde límites de grano ricos en soluto. Un tratamiento de homogeneización a $325 \mathrm{C}$ durante 50 horas seria al menos lo adecuado para lograr la homogeneización completa de aleación en lo referente a la morfología de la microestructura y a la microquímica de la aleación.

Se observa en los distintas publicaciones revisadas la atención puesta en el estudio del efecto del tratamiento térmico de homogeneizado en la ductilidad, para diferentes materiales. El fin de estos investigadores es lograr un aumento en la capacidad de procesamiento del material. Por esta razón, en este trabajo se estudia el efecto del tratamiento térmico de homogeneizado sobre la ductilidad del material, en condición "como coladas", con la finalidad de mejorar la trabajabilidad.

\section{EXPERIMENTAL}

La aleación fue producida de hornos de fosa, con crisoles de grafito de $200 \mathrm{~kg}$. Se realizaron adiciones de $1 \mathrm{~kg}$ de fundente con el objetivo de proteger el baño líquido de la atmósfera circundante. Las probetas escogidas para los diferentes ensayos fueron preparadas a partir de un lingote de CuNiSiCr, colados en coquilla refrigerada y algunas de ellas, fueron seccionadas en cubos de dimensiones $2 \times 2 \times 3 \mathrm{~cm} 3$. La tabla 1 presenta la composición obtenida de la aleación utilizada para este trabajo experimental.

Tabla 1: Composición de la aleación $\mathrm{CuNiSiCr}$

\begin{tabular}{|l|l|}
\hline elemento & peso $\%$ \\
\hline $\mathrm{Ni}$ & 2.03 \\
\hline $\mathrm{Cr}$ & 0.1 \\
\hline $\mathrm{Si}$ & 0.8 \\
\hline $\mathrm{Cu}$ & resto \\
\hline
\end{tabular}

La preparación metalográfica se realizo a través de un lijado y pulido final hasta un tamaño de partícula de $1 \mu \mathrm{m}$, con alúmina en solución acuosa. Posteriormente fueron lavadas con agua-alcohol y secadas. Para realizar la evaluación microestructural se utilizaron técnicas metalográficas convencionales. El reactivo utilizado para revelar los detalles microestructurales fue dicromato de potasio. Se realizaron los ensayos de homogeneización en un horno tubular (CARBOLITE) con atmosfera controlada, a 850,950 y $1050^{\circ} \mathrm{C}$ con tiempos de mantenimientos de $0,1,5$ y 6 hs y un posterior doble envejecido, según patente. (Edens, Ingerson., 1980).

Para estudiar la trabajabilidad se utilizaron las medidas de ductilidad obtenidas de ensayos de tracción. Estos ensayos fueron realizados a temperatura ambiente, con una velocidad de deformación de $0,1 \mathrm{~cm} /$ minuto, en una maquina de tracción (INSTRON). Para tal fin, se confeccionaron probetas equivalentes de $25 \mathrm{~mm}$ de longitud de referencia y $6.25 \mathrm{~mm}$ de diámetro, de acuerdo con la norma ASTM E8. La determinación de las fracciones de segundas fases presentes en todas las condiciones, se realizaron sobre micrografías de múltiples cortes de probetas en todas las condiciones de estudios. Para la evaluación de las fracciones se utilizo un software libre de análisis de imágenes (IMAGE J) y un microscopio óptico convencional (LEICA). Cada punto de los gráficos representa el promedio de tres valores. 


\section{RESULTADOS Y DISCUSION}

\section{Microporosidad}

La mayoría de las aleaciones, entre la que se cuentan a las aleaciones base cobre, exhiben el fenómeno de microporosidad (Bower y Granger 1988). Este fenómeno se ve favorecido si la aleación exhibe grandes cambios de volumen durante la solidificación, o cuando las dendritas formadas presentan una gran extensión. La microporosidad desmejora las propiedades mecánicas de cualquier material, de modo que es imperioso su control. Si bien en el proceso de fusión de las aleaciones de cobre se desoxida y se desgasifica el metal liquido, la humedad propia de los moldes también genera poros. La Fig.1 presenta una micrografía de la aleación CuNiSiCr, en condición de colada. En ella se observan poros producidos por gases atrapados durante el proceso de solidificación, los cuales están, en menor medida, dispersos en la matriz, pero se observan mayoritariamente en los límites de grano. Ellos actúan como centros de concentración de tensiones cuanto el material es sometido a deformaciones en frío en los procesos de conformación y reducen la sección efectiva transversal del material, conllevando a la disminución de propiedades, entre ellas la ductilidad. Según Verhoeven (1987), la microporosidad encontrada en granos equiaxiales en materiales susceptibles a la microporosidad, colados en coquillas, se encuentra en el orden de $25 \mu \mathrm{m}$ y la microporosidad hallada en granos columnares del orden de 5-10 $\mu \mathrm{m}$. En nuestro estudio fueron encontrados poros en el rango de 10-15 $\mu \mathrm{m}$ en granos equiaxiales y menores que $10 \mu \mathrm{m}$ en granos columnares.

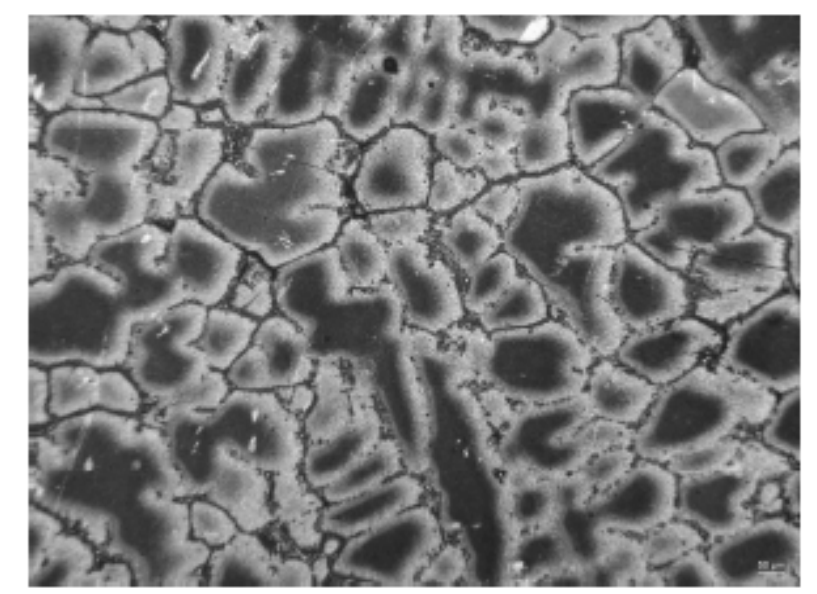

Fig. 1: estructura de colada de la aleación CuNiSiCr. Se observan poros y segundas fases (75x).

\section{Tamaño de grano}

En general, se entiende que conforme disminuye el tamaño de grano aumenta la resistencia del metal. La relación de Hall-Petch muestra que la resistencia es proporcional al reciproco de la raíz cuadrada del diámetro del grano. Sin embargo para metales colados no siempre es cierto que la resistencia mejora con la disminución del tamaño de grano, pues puede alcanzarse un menor tamaño de grano pero con un aumento en la microporosidad, o que esta se vuelva del tipo en capas, o que se aumente el porcentaje de segunda fases o que se aumente el espaciamiento dendrítico. Si alguno de estos efectos dañinos acompaña la disminución del tamaño de grano no se lograra una mejora en las propiedades mecánicas (J.D.Veroeven.1987).

En nuestra aleación de estudio, aquellas probetas homogeneizadas a $850^{\circ} \mathrm{C}$, con distintos tiempos de mantenimiento, no evidenciaron crecimiento de grano (con tamaños entre 350-550 $\mu \mathrm{m}$, aproximadamente), comparando las dos condiciones extremas a $850^{\circ} \mathrm{C}$. Esta situación es, probablemente, producida por la restricción al crecimiento de grano impuesto por el alto número de partículas de segunda fase anclando el límite. Ya en probetas homogeneizadas a $1025^{\circ} \mathrm{C}$, para distintos tiempos de mantenimiento, se observo la dilución de los precipitados en límite de grano, una evolucion de sus contornos hacia formas mas regulares y un aumento del tamaño de grano (con 
tamaños entre $400-700 \mu \mathrm{m}$, aproximadamente). Los limites de grano que conservaron precipitados, se mostraron con bajo crecimiento.

\section{Superficies de fractura}

En la actualidad existe suficiente evidencia bibliográfica acerca del mecanismo de fractura en las aleaciones dúctiles. El desarrollo de la fractura comienza con la nucleación de pequeñas grietas en las interfaces entre partículas de impurezas (inclusiones de óxidos o segundas fases en la microestructura) - matriz; y seguidamente, la deformación plástica produce cavidades que crecen y de este modo se propaga la fisura a través del material, finalizando en la fractura total del material (Brick et al., 1965).

En las Figs. 2 y 3 se presentan las micrografías de las superficies de fractura ocurridas en probetas de tracción producidas a partir de aleación CuNiSiCr, homogeneizadas a $850^{\circ} \mathrm{C}$ a diferentes tiempos de mantenimiento. Se advierte una gran diferencia en el modo de fractura. En la Fig. 2 la fractura se ha desarrollado siguiendo la trayectoria del límite de grano y no se observa coalescencia de huecos próximos a la superficie de fractura. Además, el ataque no ha puesto de manifiesto sistemas de deformación. Si bien la mayoría de las aleaciones base cobre presentan comportamientos dúctiles a la hora de sufrir una fractura a temperatura ambiente, bajo ciertas circunstancias pueden fallar de modo frágil; en estos casos la propagación de la grieta sigue la trayectoria de los limites de grano debilitados por la presencia de poros, inclusiones de óxidos y partículas de segunda fase. Las causas para este tipo de fractura son, mayoritariamente, de difícil determinación, pero en la aleación en estudio se observo la presencia significativa de partículas de segunda fase en los limites de grano y poros de gases. De modo que, la aleación CuNiSiCr, homogeneizada a $850^{\circ} \mathrm{C}$ sin tiempo de mantenimiento, sufre un tipo de fractura denominado "fractura frágil intergranular".

En la Fig. 3, se presenta un modo de comportamiento del material diferente, a través de su modo de fractura. La micrografía muestra la presencia de bandas de deslizamiento, claramente resaltadas por el ataque en todos los granos próximos a la superficie de fractura. Se observa también la coalescencia de huecos, lo que en alguna región en particular ya se ha trasformado en un cavidad. Este tipo de fractura tiene todas las características de una fractura dúctil, donde el esfuerzo que desarrolla la fractura es el cizallamiento. Además se advierte una gran deformación plástica antes de la rotura (estricción) y en consecuencia se produce el alargamiento de los granos, como es mostrado en la Fig. 3.

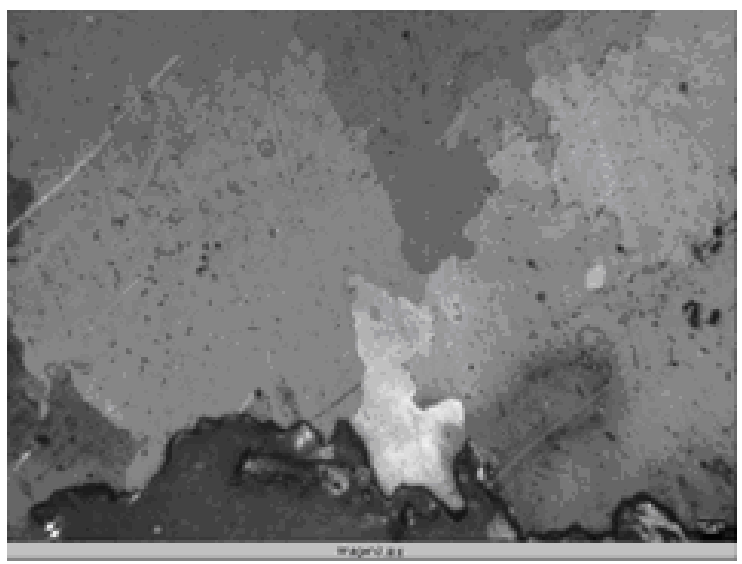

Fig. 2: Propagación de la fisura siguiendo la trayectoria del límite de grano (x75) -

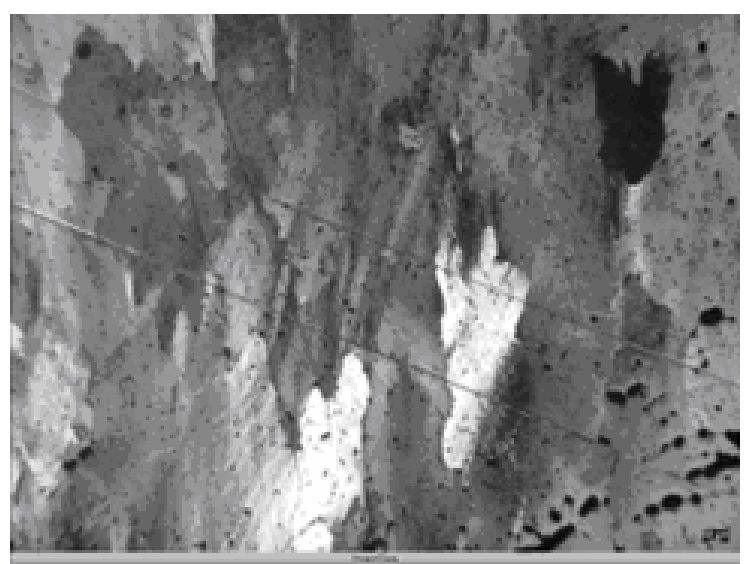

Fig. 3: Fluencia de los granos próximos a la superficie de fractura y la coalescencia de huecos

En las probetas homogeneizadas a $1025^{\circ} \mathrm{C}$ y con tiempos de mantenimiento de 6 hs, el comportamiento de microestructura fue también dúctil. En la Fig.4 se observa la presencia de bandas de deslizamiento y coalescencia de huecos próximos a la superficie de fractura. La propagación de la fisura parece seguir la trayectoria de los limites de grano, siendo entonces una fractura dúctil intergranular. 


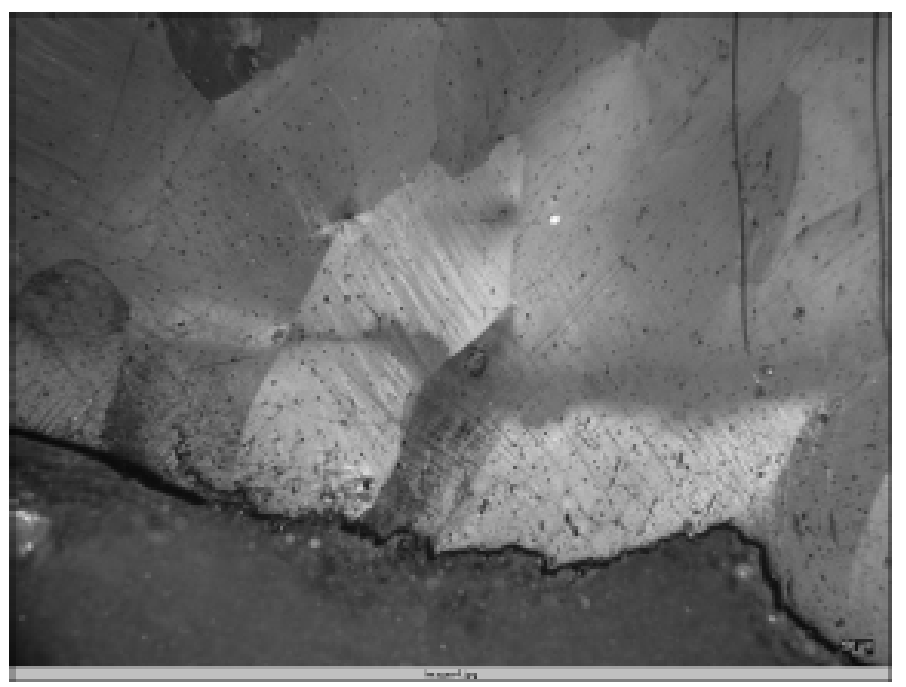

Fig. 4- Propagación intergranular de la fisura; hay coalescencia de huecos y bandas de deslizamiento. (x75)

\section{Ensayos de tracción}

Uno de los desafíos en el diseño de aleaciones es lograr un aumento de la resistencia a través de la precipitación de una segunda fase en forma fina y dispersa. Al mismo tiempo, el incremento de esta propiedad debe permitir un flujo plástico en las puntas de las grietas y evitar la nucleación o coalescencia de huecos durante el flujo de material. Sin embargo es común observar, por ejemplo en los aceros templados y revenidos que un aumento de la resistencia produce una reducción en la ductilidad, disminuyendo así la trabajabilidad del material. Este aumento de la resistencia se explica por la inmovilización de las dislocaciones y, como la ductilidad requiere de dislocaciones móviles, surge entonces una incongruencia natural entre la resistencia y la ductilidad.

En la Fig. 5 se presenta el efecto de la temperatura y el tiempo de homogeneizado en función de la trabajabilidad de la aleación $\mathrm{CiNiSiCr}$, para probetas homogeneizadas a 850,950 y $1025^{\circ} \mathrm{C}$ y para tiempos de mantenimiento de $0,1.5$ y $6 \mathrm{hs}$. La Fig.5(a) muestra un comportamiento lineal entre la ductilidad y el tiempo de homogeneizado para todas las condiciones de ensayos. Sin embargo, los valores de resistencia en la Fig. 5(b), muestran un comportamiento dispar en las temperaturas de ensayo. Para probetas homogeneizadas a $850^{\circ} \mathrm{C}$, con distintos tiempos de mantenimiento, se observa un comportamiento similar a los aceros templados y revenidos: para un aumento de resistencia se reduce la ductilidad. Para muestra homogeneizadas a $950^{\circ} \mathrm{C}$, se observa que la ductilidad aumenta pero la resistencia se muestra inalterable con el tiempo de homogeneizado.
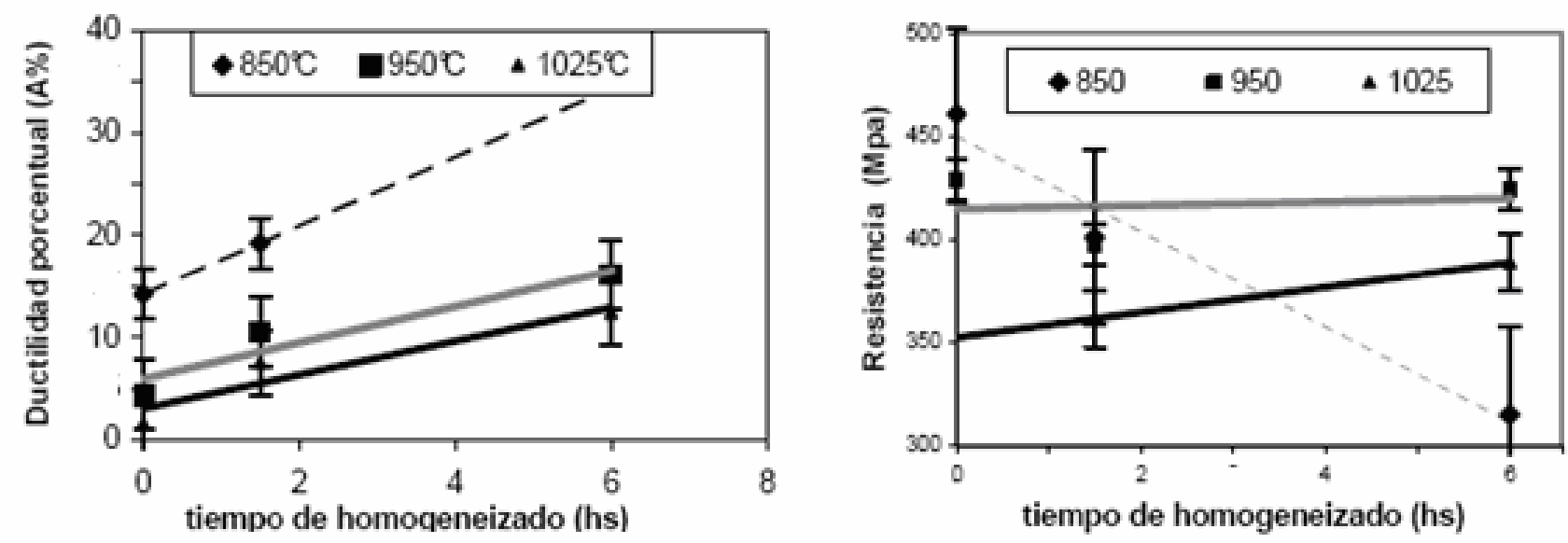

Fig.5.- Relación entre propiedades y temperatura-tiempos de homogeneizado 
Ya en las probetas homogeneizadas a $1025^{\circ} \mathrm{C}$ parece suceder todo lo contrario. Se observa que, a medida que aumenta la ductilidad, lo hace la resistencia. Si bien esta situación parece inusual, hay un variado grupo de aleaciones que se comportan en forma similar, como por ejemplo las fundiciones esferoidales o en aquellas aleaciones donde el tratamiento térmico de homogeneizado disuelve los precipitados. Esta disolución de precipitados lleva a un fortalecimiento de la matriz por endurecimiento por solución sólida, ocasionando un aumento de resistencia. Es evidente entonces, que el control de las segundas fases en la aleación CuNiSiCr asume una gran importancia a la hora de optimizar las propiedades.

\section{Segundas fases}

Las propiedades y características estructurales, tanto de la matriz como de las segundas fases presentes en cualquier material, afectan directamente su ductilidad. El efecto que produce una segunda fase sobre las propiedades mecánicas es siempre significativo y tiene relación directa con la trabajabilidad del material. Singh et al., (1968) estudiaron la influencia que producen segundas fases en las propiedades mecánicas de una aleación de aluminio 7075 . Sus resultados muestran que se genera un aumento significativo de la ductilidad conforme se elimina la segunda fase.

Observando las micrografías de la Fig.6 provenientes de probetas extraídas de material homogeneizado a $850^{\circ} \mathrm{C}, 950^{\circ} \mathrm{C}$ y $1025^{\circ} \mathrm{C}$ se pueden ver las diferentes formas y distribuciones que pueden formar los precipitados como resultado de los tratamientos térmicos de homogeneizado. Las micrografías presentan segundas fases en las regiones interdendriticas y limites de grano. Estas fases son, fundamentalmente, inclusiones de óxidos y $\mathrm{Cr}_{3} \mathrm{Si}$. (Rdzawski, Stobrawa.,1996).

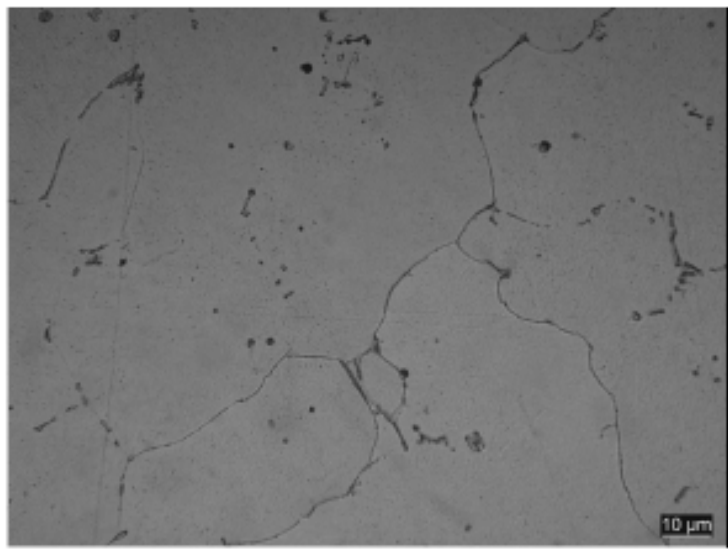

(a) $850^{\circ} \mathrm{C}, 0 \mathrm{hs}$

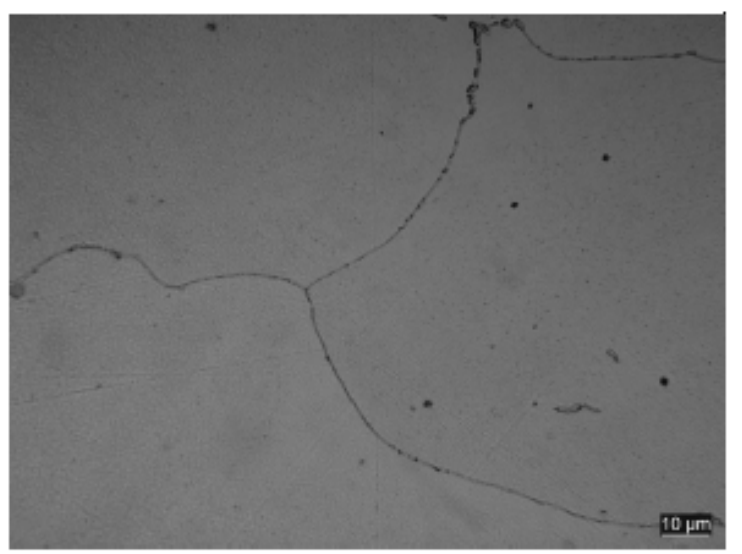

(c) $950^{\circ} \mathrm{C}, 6 \mathrm{hs}$

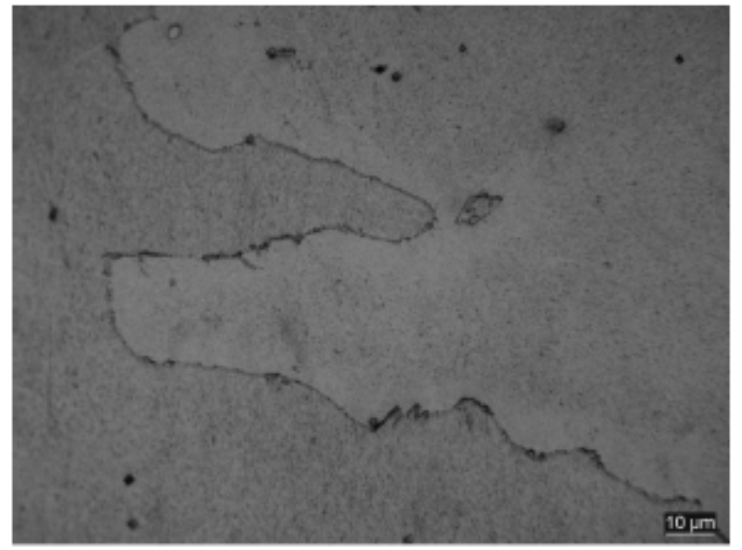

(b) $850^{\circ} \mathrm{C}, 6 \mathrm{hs}$

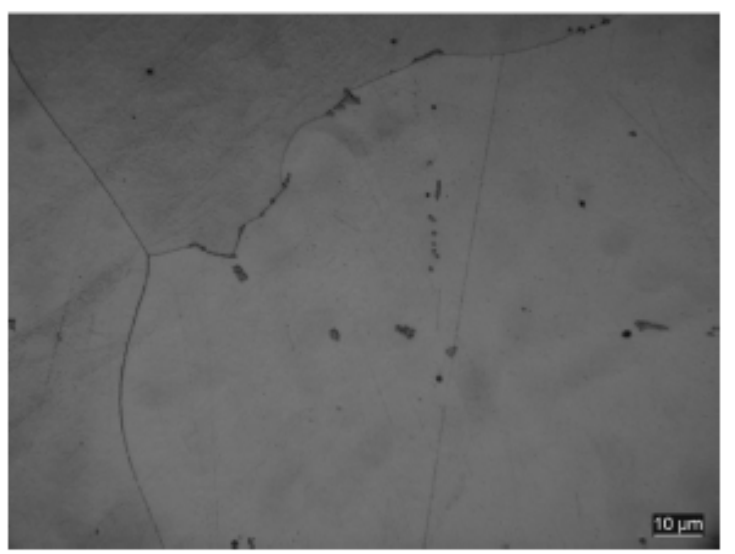

(d) $1025^{\circ} \mathrm{C}, 6 \mathrm{hs}$

Fig. 6.- Los cambios en la forma y cantidad de precipitados de la aleación CuNiSiCr envejecidas a) $850^{\circ} \mathrm{C}$, sin tiempo de mantenimiento b) $850^{\circ} \mathrm{C}$, 6hs de mantenimiento c) $950^{\circ} \mathrm{C}$, $6 \mathrm{hs}$ de mantenimiento d) $1025^{\circ} \mathrm{C}$. 6hs de mantenimiento (1000x.) 
La Fig.6(a) corresponde a una micrografía de una probeta calentada a $850^{\circ} \mathrm{C}$ y enfriada en agua inmediatamente, lo que significa sin tiempo de mantenimiento. Se observa una gran cantidad de segundas fases en límite de grano, de formas irregulares y también en el interior del grano y estas aun perduran en los límites después de haber mantenido la muestra por espacio de 6 hs (Fig.6-b). Todas las medidas de tamaño de grano arrojaron valores idénticos, lo cual nos lleva a inferir que promueven el anclaje del grano.

Las micrografías ópticas siguientes muestran la evolución, en forma, tamaño y distribución de las segundas fases. Sus morfologías son mas regulares y se presentan de manera discontinua en el límite de grano. Las muestras homogeneizadas a $950^{\circ} \mathrm{C}$ y $1025^{\circ} \mathrm{C}$ presentan diferencias con respecto a la condición de $850^{\circ} \mathrm{C}$. Además de observarse un aumento de la regularidad del límite de grano producto de la disolución de precipitados, también hay una considerable disminución de la cantidad de precipitados. Esta transformación es desarrollada por el proceso de difusión, eliminando también la segregación. Ahora, la menor cantidad de precipitados genera dos efectos: por un lado endurece la matriz, como lo demuestra el aumento de resistencia y por otro permite un tamaño de grano mayor, lo cual promueve más susceptibilidad a la fractura intergranular.

\section{Relación entre ductilidad y segundas fases}

Las segundas fases juegan siempre un rol dominante en la fractura dúctil de aleaciones termotratables. Se convierten entonces en factores de gran importancia, su naturaleza, forma y distribución en la matriz, así como su fracción en volumen (Hosford., 1993). Durante la solidificación de la aleación CuNiSiCr se produce una microestructura de dos fases debido a la segregación dendrítica. Esta microestructura disminuye sensiblemente las propiedades mecánicas, entre ellas la ductilidad, por lo tanto disminuye la trabajabilidad.

Una de las formas de caracterizar la cantidad de microsegregación de una aleación como colada, es determinar la fracción de segunda fase presente para las diferentes condiciones de tratamientos térmicos y relacionarla de algún modo con las propiedades del material. La interacción entre ductilidad y segundas fases se pone en evidencia en el gráfico de la Fig.7. Se observa claramente que la ductilidad es función directa tanto del tratamiento térmico como de la temperatura de homogeneizado.

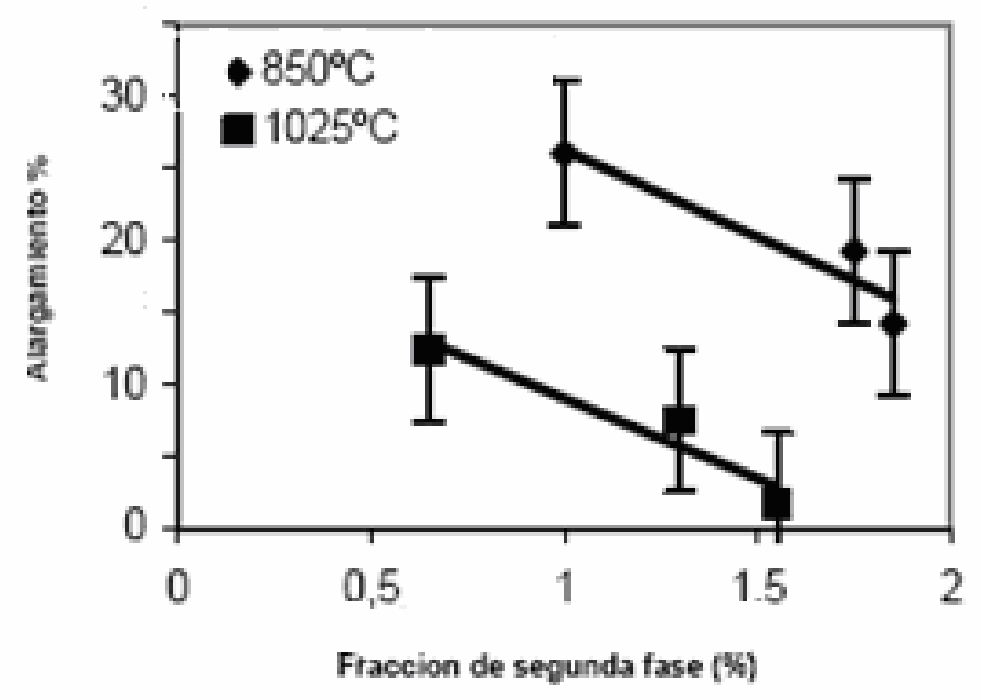

Fig. 7.- Relación entre trabajabilidad y segundas fases.

\section{CONCLUSIONES}

Después del estudio realizado en la aleación CuNiSiCr, se observa que el tratamiento térmico de homogeneizado afecta la microestructura de la aleación CuNiSiCr. Se demuestra que los parámetros 
temperatura y el tiempo de homogeneizado son vitales y afectan la trabajabilidad. El tiempo de homogeneizado parece tener gran importancia en las probetas homogeneizadas a $850^{\circ} \mathrm{C}$ durante 6 horas, ya que modifica las condiciones de falla de fractura de frágil a dúctil, parámetro que presenta una relación directa con la trabajabilidad.

Por otro lado, un aumento en la temperatura de homogeneizado desmejora la trabajabilidad debido al aumento exagerado del tamaño de grano. En probetas en condición de como coladas, las partículas de segunda fase son grandes y de forma irregular. Después de la aplicación de los tratamientos térmicos, estas modifican su morfología a redondeadas y se presentan de manera discontinua en límites de grano.

Los ensayos de tracción muestran un aumento en la ductilidad cuando aumenta el tiempo de homogeneizado. Del estudio realizado se puede inferir que las condiciones optimas para el tratamiento térmico de homogeneizado es $850^{\circ} \mathrm{C}$ durante 6 horas. Se concluye que el tratamiento térmico de homogeneizado es una herramienta adecuada para aumentar la trabajabilidad de un material en condición cómo colado.

\section{AGRADECIMIENTOS}

los autores agradecen a los Sres Leonello y Flavio Marchiori de la empresa COOPERSOL SA, por suministrar el material para este estudio.

\section{REFERENCIAS}

Apello, M y P. Fenici, Solution Heat Treatment of a Cu-Ni-Zr Alloy, Materials Sc. and Eng A: 102, 6975 (1988)

ANSI/ASTM E8 -79a, Tension testing of metallic materials,1039-1058, 100 Barr Harbor Drive, PO Box C700, West Conshohocken, PA, 19428-2959, USA (1979)

Bhargava, M K y S. Bhan, Studies on Age Hardening Cu-Ni-Si-Mg and Cu-Ni-Si-Cr alloys, ,Z.Metallkde Bd: 63, H.3 (1972)

Bower, T F y D.A Granger, Solidification Structure of Copper Alloys Ingots, Metals Handbook: 9, 9th Edition, 637-645 (1989)

Brick, R.M, Gordon y R.B, Phillips, Structure and properties of metals, 3th Ed, McGraw-hill, NY, United States of America (1965)

Edens, W. W y Q.F Ingerson, Copper-nickel-silicon-chromium alloy having improved electrical conductivity, U.S.Patent N 4191601, current US Class 148/686, 4 de Marzo de 1980 (1980)

Dang-sheri' MA, ZHOU jian' , CHEN Zai-zhi' , ZHANG Zhong-kan' ,CHEN Qi-an' y LI De-hui, Influence of Thermal Homogenization Treatment on Structure and Impact Toughness of H13 ESR Steel, Journal of iron and steel research international, 16(5): 56-60, (2009).

Dieter, G.E, Forming and Forging, ASM Handbook: 14 (1996)

Hosford, W. F y R. M. Caddell. Metal Forming Mechanics and Metallurgy, 2nd edition, PTR PrenticeHall a Pearson Education Company, Upper Saddle River, NJ 07458 (1993)

Jing Zhang, Rulin Zuo, Youxing Chen, Fusheng Pan, Xiaodong Luo, Microstructure evolution during homogenization of a type Mg-Zn-Al alloy, Journal of Alloys and Compounds 448 316-320, (2008)

Obrutsky, D, Serrante, M, y O. Wortman, Propiedades de una Aleación de Cobre de alta resistencia al ablandamiento y alta conductividad, Informe N¹46, CNEA (1965) 
Rdzawski, Z y J. Stobrawa, thermomechanical processing of Cu-Ni-Si-Cr-Mg alloy, Materials A science and Technology: 9, 142-149 (1993)

Saarivirta, M.J y P.P Taubenblat, Some High-Temperature Properties of Copper-Zirconium and Copper-Chromium High-Conductivity Alloys, Trans. Of Metallurgical Society of AIME: 218, 935-939 (1960)

Saarivirta, M.J., High Conductivity Copper Rich Cu-Zr Alloys, Trans. Of Metallurgical Society of AIME: 218, 431-437 (1960)

Flemings M.C., solidification processing, pp 364, McGraw-Hill, Inc, USA (1974)

Sokolowski, J.H, Sun, X-C, Byczynsky, G, Northwood, D.O, Thomas, D.E y A. Esseltine, The removal of copper-phase segregation and the subsequent improvement in mechanical properties of the cast 319 aluminium alloys by a two stages solution heat treatment, Journal of materials Processing Technology: 53, 385-392 (1995)

Susuki, S, Shibutani, N, Mimura, K, Isshiki, M y Y. Waseda, Improvement in strength and electrical conductivity of CuNiSi alloys by aging and cold rolling, Journal of alloys and Compounds: 417,116$120(2006)$

Tewari, R.A y Bhan, Effects of small alloying additions on the ageing behavior of a copper-nickelsilicon alloy. Transaction of the Indian institute of metals: 211-216 (1964)

Totik, Y, Sadeler, R, Kaymaz, I y M. Gavgali, The effect of homogenisation treatment on cold deformations of AA 2014 and AA 6063 alloys, Journal of Materials Processing Technology: 147(1), 60-64 (2004)

Verhoeven, J.D., Fundamentos de metalurgia física, Ed. Limusa, John Wiley and Sons Inc, Mexico DF (1987)

Zhang Wei, Liu Yong, Huang Jinsong, Liu Bin, He Yuehui, Huang Boyun, Optimization of Microstructure and Elimination of $\beta(B 2)$ Phase in As-Cast Ti-Al-Nb-W-B Alloy, Rare Metal Materials and Engineering Volume 38, Issue 10, (2009) 